\title{
A Retrospective Investigation on Electric Bidet Use as a Possible Cause of Anal Incontinence
}

\author{
Akira Tsunoda and Hiroshi Kusanagi \\ Department of Gastroenterological Surgery, Kameda Medical Center, Kamogawa, Japan
}

\begin{abstract}
Objectives: The present study aimed to explore whether symptoms of anal incontinence (AI) in patients who used electric bidet toilets to clean the anus may improve after discontinuing bidet use.

Methods: Fifty-three patients with AI who habitually used the bidets before or after defecation and were examined between June 2019 and September 2020 were included in this retrospective study. Questionnaires on Likert-scaled items that assessed bidet use were administered at baseline. The sum of all points was regarded as the "bidet use score". The patients were instructed to discontinue bidets until subsequent examination. Incontinence severity was documented using the fecal incontinence severity index (FISI) score.

Results: Follow-up data were available for 49 patients (92\%). Of those, 43 had fecal incontinence and 6 had only mucus discharge at baseline. The median duration between the baseline and follow-up was 4 weeks. The median FISI score was significantly reduced at the follow-up [baseline vs. follow-up: 15 (range: 3-43) vs. 10 (range: $0-43$ ); $P<0.0001]$. The incidence of fecal incontinence was significantly lower at the follow-up than at the baseline $(59 \%$ vs. $88 \%, P=0.003)$. A higher maximum squeeze pressure and the absence of associated factors that may cause AI (such as rectoanal intussusception and/or rectocele, mucosal prolapse, and previous anorectal surgery) were significantly associated with a reduction of at least $50 \%$ in the FISI scores at follow-up; however, this was not observed for the bidet use score.

Conclusions: Our findings suggest that electric bidet use is a possible cause of AI.
\end{abstract}

\section{Keywords}

electric bidet toilet, anal incontinence, Japanese

\section{Introduction}

Electric bidet toilets use is a growing global trend[1]. These are automatic devices that deliver a jet of water to clean the anus after defecation. Ever since their introduction in the Japanese market in 1967, Japanese-made electric bidet toilets have steadily gained popularity, and presently, around $80 \%$ of the Japanese households have bidet toilets[2]. Bidets have been developed to incorporate different functions to improve user comfort. Today, users can select the preferred force, thickness, and temperature of the water jet. 
Table 1. The Bidet Use Score Questionnaire.

\begin{tabular}{lcccc}
\hline \multirow{2}{*}{ Variables } & \multicolumn{4}{c}{ Score } \\
\cline { 2 - 5 } & 0 & 1 & 2 & 3 \\
\hline Frequency of washing & $\leq 3$ times/week & $4-7$ times/week & $2-3$ times/day & $\geq 4$ times/day \\
Force of water jet & weak & moderate & strong & \\
Thickness of water jet & thick & thin & & $\geq 31 \mathrm{sec}$. \\
Duration of washing & $<5 \mathrm{sec}$. & $6-10 \mathrm{sec}$. & $11-30 \mathrm{sec}$. & \\
\hline
\end{tabular}

Therefore, the aim of the study was to explore whether the symptoms of AI in patients who used electric bidet toilets to clean the anus may improve after discontinuing bidet use.

\section{Methods}

Between June 2019 and September 2020, 69 patients with symptoms of AI were examined by one physician at a proctology clinic in Kameda Medical Center and prospectively registered in a pelvic floor database. Of these, 53 patients (77\%), who habitually used electric bidet toilets before or after defecation were included in this retrospective study. The remaining 16 patients who did not use bidets were excluded from the study. A structured interviewer-led questionnaire consisting of four two- to four-point Likert-scaled items (Table 1) was administered at the initial consultation for assessing how the patients used bidets to clean the anus. Each of the Likert-scaled items had two or four possible answers with scores ranging from 0 (less active) to 3 (more active use). The "bidet use score" was the sum of all points and could be maximum 9 points. The included patients were instructed to discontinue bidet use until subsequent examination by the same physician. Other instructions on treating AI or medical treatment for AI were not given during the follow-up period. At the follow-up, they were confirmed to have discontinued bidet use. Incontinence severity was documented using the fecal incontinence severity index (FISI) score[4]. The patients were advised to undergo defecography and laboratory tests for assessing anal sphincter function. Written informed consent was obtained from all patients. This study was approved by the Ethical Committee of the Kameda Medical Center (approval number: 20-091)

\section{Defecography}

A standardized defecography technique was used to evaluate the patients' pelvic and rectal function, and proctograms were evaluated using the criteria proposed by Shorvon et al.[5]. Briefly, rectoanal intussusception was diagnosed when images obtained during maximal straining defecation indicated that the apex of the rectal intussusception impinged on the internal anal orifice or was intra-anal. A rectocele greater than $2 \mathrm{~cm}$ in diameter was regarded as ab- normal; the size was calculated in the anterior-posterior dimension by measuring the distance between the actual most ventral part of the anterior rectal wall and an extrapolated line indicating the expected position of the rectal wall[6].

\section{Laboratory studies on anal sphincter function}

Patients were examined in the left lateral position and no bowel preparation was undertaken. The anal pressure was measured with a catheter-tip pressure transducer. The capacity of the rectum was measured using a balloon, with its lower extremity placed at a distance of $5 \mathrm{~cm}$ from the anal verge. Rectal perception of distention was examined through continuous and slow air insufflation into the balloon, and the volume for the desire to defecate and maximum tolerated volume were measured.

\section{Statistical analysis}

The data were analyzed using SPSS version 11.0 for Windows (SPSS Japan Institute, Tokyo, Japan). $P<0.05$ was considered to indicate statistical significance. Quantitative data were expressed as medians and ranges, except for the scores on each of the FISI component, which were presented as means and 95\% confidence intervals. Unpaired data were analyzed using the Mann-Whitney $U$-test, whereas pairs of FISI scores were compared using the Wilcoxon signed-rank test. Categorical variables were compared using the $\chi^{2}$ test or the Fisher's exact test. Correlations between continuous variables were analyzed using Pearson's correlation coefficient. Substantial improvement in AI was defined as a reduction of at least $50 \%$ in FISI scores. The primary objective was to assess the changes in FISI scores after discontinuing bidet use. The secondary objective was to explore correlations between a substantial improvement in AI after discontinuing bidet use and variable demographic, manometric, and bidet use parameters.

\section{Results}

The median age of the 53 patients included (comprising 27 women and 26 men) was 71 years (range: $35-88$ years). The median FISI and bidet use score were 16 (range: 3-43) and 4 (range: 1-9), respectively. There was no significant correlation between the two scores $(P=0.92)$. Although 
Table 2. Number of Patients Who Reported Bidet Using Variables.

\begin{tabular}{lcccc}
\hline & $\begin{array}{c}\text { Total } \\
(\mathrm{n}=53)\end{array}$ & $\begin{array}{c}\text { Men } \\
(\mathrm{n}=26)\end{array}$ & $\begin{array}{c}\text { Women } \\
(\mathrm{n}=27)\end{array}$ & $P$ \\
\hline $\begin{array}{l}\text { Frequency of washing } \\
\quad 3 \text { times/week }\end{array}$ & 5 & 1 & 4 & 0.42 \\
$\quad$ 4-7 times/week & 19 & 11 & 8 & \\
$\quad$ 2-3 times/day & 21 & 11 & 10 & \\
$\quad$ 4 times/day & 8 & 3 & 5 & \\
Force of water jet & & & & \\
$\quad$ weak & 18 & 8 & 10 & 0.78 \\
$\quad$ moderate & 19 & 9 & 10 & \\
$\quad$ strong & 16 & 9 & 7 & \\
Thickness of water jet & & & & \\
$\quad$ thick & 11 & 2 & 9 & 0.04 \\
$\quad$ thin & 42 & 24 & 18 & \\
Duration of washing & & & & \\
$\quad<5$ sec. & 15 & 6 & 9 & 0.47 \\
$\quad$ 6-10 sec. & 21 & 9 & 12 & \\
$\quad$ 11-30 sec. & 12 & 8 & 4 & \\
$\quad$ 31 sec. & 5 & 3 & 2 & \\
Median (range) bidet use score & $4(1-9)$ & $5(2-9)$ & $4(1-7)$ & 0.15 \\
\hline
\end{tabular}

Table 3. Characteristics of Patients with Anal Incontinence Who Were Followed-up.

\begin{tabular}{lc}
\hline Anal incontinence, $n$ & 49 \\
Fecal incontinence & 43 \\
Mucus discharge alone & 6 \\
Age, years & $71(35-88)$ \\
Female, $n(\%)$ & $23(47)$ \\
Bidet use score & $4(1-9)$ \\
Fecal Incontinence Severity Index score & $15(3-43)$ \\
Duration of discontinuing a bidet use, weeks & $4(2-20)$ \\
Associated disorders, $n$ & 22 \\
Rectoanal intussusception and/or rectocele & 9 \\
Mucosal prolapse & 4 \\
Previous anorectal surgery & 3 \\
Functional defecation disorders & 2 \\
Cauda equina disorder & 1 \\
Rectal carcinoma & 1 \\
Ulcerative colitis & 1 \\
Previous radiotherapy & 1 \\
\hline
\end{tabular}

Values are median (range), otherwise indicated.

men generally used a thinner water jet as compared to women, the frequency of washing, force of the water jet, and duration of washing did not differ significantly between the two sexes (Table 2).

Follow-up data were available for 49 patients (92\%) who were confirmed to have discontinued bidet use. The median duration between the baseline and follow-up was 4 weeks (range: 2-20 weeks). Twenty-two patients (45\%) had associated disorders (Table 3). Twenty-three of the 49 patients (47\%) underwent defecography, whose findings included
Table 4. Number of Patients with Fecal Incontinence.

\begin{tabular}{lcccl}
\hline & Evaluated patients & Baseline & Follow-up & \multicolumn{1}{c}{$P^{*}$} \\
\hline Total & 49 & $43(88 \%)$ & $29(59 \%)$ & 0.003 \\
Men & 26 & $22(85 \%)$ & $13(50 \%)$ & 0.02 \\
Women & 23 & $21(91 \%)$ & $16(70 \%)$ & 0.14 \\
\hline$\chi^{2}$ test & & & &
\end{tabular}

rectoanal intussusception and/or rectocele $(n=9)$, pelvic floor dyssynergia $(n=1)$, and inadequate defecatory propulsion $(n=1)$. Furthermore, 43 of the 49 patients $(88 \%)$ had FI at the baseline, and the incidence of FI at follow-up $(59 \%)$ was significantly lower than that at the baseline $(59 \%$ vs. $88 \%$ ) (Table 4). The remaining six patients had a mucus discharge alone. The FISI scores were significantly reduced at the follow-up [median score at baseline vs. that at followup: 15 (range: $3-43$ ) vs. 10 (range: 0-43), $P<0.0001$ ] (Figure 1). A substantial improvement in AI was noted in 21 patients $(43 \%)$ after they discontinued bidet use; $14(67 \%)$ of these recovered anal continence completely (Table 5). Of the six patients with mucus discharge alone, three (50\%) recovered anal continence completely at the follow-up. When the baseline and follow-up scores for each of the FISI components were compared, it was observed that the scores of the gas and liquid stool components were significantly reduced at the follow-up $(P=0.01, P<0.0001$, respectively) (Table $6)$.

Of the 28 patients who did not show a substantial improvement in the AI after discontinuing bidet use, 17 (61\%) presented with the following factors that may result in AI: 
Table 5. Fecal Incontinence Severity Index Scores.

\begin{tabular}{lccccc}
\hline & \multirow{2}{*}{$\begin{array}{c}\text { Evaluated patients } \\
(n)\end{array}$} & Baseline & Follow-up & \multicolumn{2}{c}{ Patients with substantial } \\
\cline { 5 - 6 } & 49 & $15(3-43)$ & $10(0-43)$ & $21(43)$ & improvement $^{\mathrm{a}}, n(\%)$ \\
\hline Total & 26 & $13(3-37)$ & $6.5(0-20)$ & $14(54)$ & $<0.0001$ \\
Men & 23 & $20(3-43)$ & $13(0-43)$ & $7(30)$ & 0.0001 \\
Women & 23 &
\end{tabular}

${ }^{\mathrm{a}}$ Reduction of at least 50\% in Fecal Incontinence Severity Index Score after discontinuing bidet toilet use,

${ }^{\mathrm{b}}$ Versus baseline (Wilcoxon signed rank test)

Values are median (range), otherwise indicated.

Table 6. Baseline and Follow-up Scores for Each of the FISI Components.

\begin{tabular}{lccc}
\hline & baseline $(\mathrm{n}=49)$ & Follow-up $(\mathrm{n}=49)$ & $P^{*}$ \\
\hline Gas & $3.9(2.5-5.4)$ & $2.6(1.4-3.8)$ & 0.01 \\
Mucus & $2.0(1.0-3.1)$ & $1.6(0.5-2.6)$ & 0.24 \\
Liquid stool & $8.4(6.7-10.1)$ & $4.5(2.7-6.3)^{\dagger}$ & $<0.0001$ \\
Solid stool & $3.0(1.5-4.4)$ & $2.6(1.2-4.0)$ & 0.50 \\
\hline
\end{tabular}

Values are presented as mean (95\% confidence interval).

FISI, Fecal Incontinence Severity Index

* Wilcoxon signed rank test; ${ }^{\dagger}$ Versus baseline

Table 7. Pearson's Correlation Coefficients between a Substantial Improvement ${ }^{\mathrm{a}}$ in Anal Incontinence and Variables.

\begin{tabular}{lcc}
\hline & Coefficients & $P$ \\
\hline Age (years) & 0.253 & 0.25 \\
Sex (men, women) & -0.236 & 0.10 \\
Bidet use scores & 0.104 & 0.48 \\
Associated disorders (no, yes) & -0.367 & 0.009 \\
Maximum resting pressure* $\left(\mathrm{cmH}_{2} \mathrm{O}\right)$ & -0.012 & 0.95 \\
Maximum squeeze pressure* $\left(\mathrm{cmH}_{2} \mathrm{O}\right)$ & 0.607 & 0.001 \\
Volume for desire to defecate* $\left(\mathrm{ml}^{2}\right)$ & 0.194 & 0.32 \\
Maximum tolerated volume* $(\mathrm{ml})$ & 0.143 & 0.47 \\
\hline a Reduction of at least 50\% in Fecal Incontinence Severity Index Score \\
after discontinuing bidet toilet use, \\
${ }^{*} n=28$.
\end{tabular}

rectoanal intussusception and/or rectocele $(n=7)$, mucosal prolapse $(n=4)$, cauda equina syndrome $(n=1)$, rectal carcinoma $(n=1)$, previous fistulotomy for an anal fistula $(n=$ $1)$, previous surgery for rectal prolapse $(n=1)$, pelvic floor dyssynergia $(\mathrm{n}=1)$, and inadequate defecatory propulsion causing overflow FI $(n=1)$. Of the 21 patients who had a substantial improvement in AI after discontinuing bidet use, five presented with the following factors that may result in AI: rectoanal intussusception and/or rectocele $(n=2)$, previous fistulotomy for an anal fistula $(n=1)$, previous radiotherapy for prostate carcinoma $(n=1)$, and ulcerative colitis $(\mathrm{n}=1)$.

Twenty-eight $(57 \%)$ of the 49 AI patients underwent a manometric study. The median maximum resting pressure and maximum squeeze pressure were $60 \mathrm{cmH}_{2} \mathrm{O}$ (range: 22-

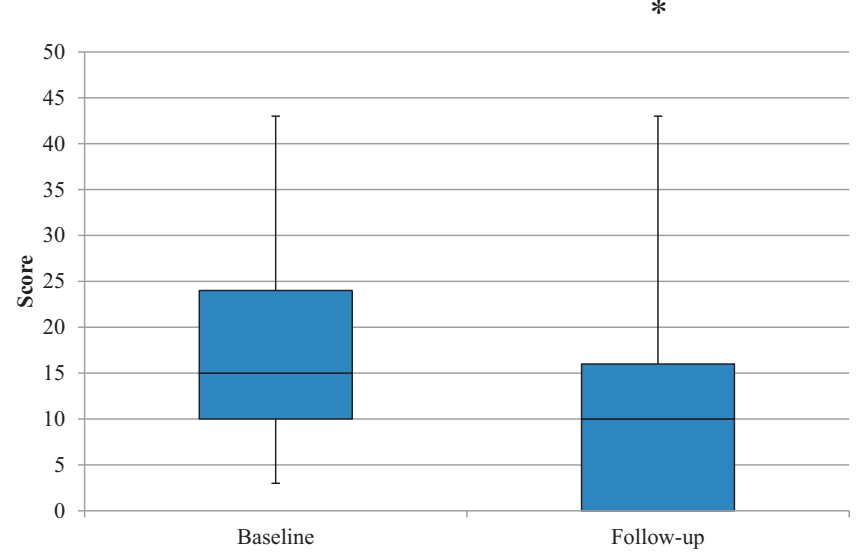

Figure 1. The fecal incontinence severity index scores at the baseline and follow-up. Boxes show median values with upper and lower quartiles. The vertical line extends from the minimum to the maximum values. $* P<0.0001$ versus the baseline (Mann-Whitney $U$-test).

$173 \mathrm{cmH}_{2} \mathrm{O}$ ) and $212 \mathrm{cmH}_{2} \mathrm{O}$ (range: 47-521 $\mathrm{cmH}_{2} \mathrm{O}$ ), respectively. The median volume for the desire to defecate and the maximum tolerated volume were $118 \mathrm{~mL}$ (range: $40-300$ $\mathrm{mL}$ ) and $233 \mathrm{~mL}$ (range: $95-300 \mathrm{~mL}$ ), respectively. The correlations between a substantial improvement in AI after discontinuation of bidet use and the variable demographic, manometric, and bidet use parameters are listed in Table 7. Absence of the associated disorders (that may cause AI) and a higher maximum squeeze pressure were significantly associated with a substantial improvement in AI. The median maximum squeeze pressure was significantly higher in patients with a substantial improvement in AI than that in patients without a substantial improvement $\left[348 \mathrm{cmH}_{2} \mathrm{O}(n=\right.$ 8) vs. $\left.194 \mathrm{cmH}_{2} \mathrm{O}(n=20), P=0.002\right]$. Furthermore, the bidet use score was not correlated with a substantial improvement in AI.

\section{Discussion}

To best of our knowledge, this is the first study demonstrating that patients with AI who used electric bidet toilets to clean the anus experienced a significant improvement in 
AI after discontinuing bidet use. Furthermore, patients with a higher maximum squeeze pressure and absence of the associated disorders that may cause AI (such as rectoanal intussusception and/or rectocele, mucosal prolapse, and previous anorectal surgery) experienced a substantial improvement in AI after discontinuing bidet use; however, this was not observed for the bidet use score.

There have been no reports on the effects of bidet toilet use on AI to date. Our previous questionnaire-based survey indicated that 2,724 respondents used bidet toilets before or after defecation, and 156 of these experienced FI one or more times a month after leaving the lavatory[3]. The responders reported that they expected the bidet to aid in defecation by stimulating the anus with a jet of water. A previous study reported that patients with spinal cord injury who used a bidet before defecation as a defecation aid preferred a strong water jet, possibly because it aided defecation in the same manner as an enema with water penetrating into the rectum[7]. This, in fact, was confirmed by a previous study that reported an increase in the rectal temperature after application of warm water, suggesting that water may penetrate the rectum[8]. Therefore, it is possible that when our patients washed the anus with a bidet, water might have penetrated the rectum, especially in those with a lax anal sphincter. This may be explained by a comparison of the baseline and follow-up scores for each of the FISI components in this study: The scores of the liquid stool component were significantly reduced after discontinuation of the bidet use. Enemas induce the defecation reflex and increase bowel peristalsis with water streaming into the rectum, thereby resulting in symptoms of AI post defecation.

The FISI score has been used to assess the severity of $\mathrm{AI}[9,10]$. When one treats patients with AI, one should compute the FISI score at the baseline cautiously; otherwise, the effect of treatment on AI or a comparison of the pre- and post-FISI scores would be unreliable. In this study, a significant improvement in the AI was noted in $43 \%$ of the patients after they discontinued bidet use. When the severity of AI is estimated using not only the FISI score but also other parameters, researchers should check whether a patient with $\mathrm{AI}$ is a bidet user. If the patient uses a bidet, instead of treating AI immediately, the patient should be instructed to discontinue bidet use for a certain period of time and the severity of $\mathrm{AI}$ should be assessed again later.

In this study, patients with a higher maximum squeeze pressure and absence of the associated disorders that may cause AI experienced a substantial improvement in AI after discontinuing bidet use. Though the reason for AI improvement in patients with a higher maximum squeeze pressure is unclear, it is possible that patients who use a bidet would feel a stronger urgency of defecation due to increased bowel peristalsis induced by an enema effect, as mentioned earlier. Once bidet use is discontinued, the bowel peristalsis may stabilize, and those with a higher squeeze pressure can control a weaker urgency of defecation and hold the feces in the rectum. AI improvement in patients with no associated disorders is understandable upon considering the symptoms of associated disorders. Rectoanal intussusception and/or rectocele and mucosal prolapse are known causes of FI and/ or mucus discharge[11-13], and those with such disorders may obviously not experience a significant improvement in the AI after discontinuing bidet use. Other associated factors including the cauda equina syndrome, rectal carcinoma, and previous anorectal surgeries may also cause AI. One patient with pelvic floor dyssynergia had a lax anal sphincter, which may be caused by chronic excessive straining that leads to pudendal nerve damage.

In this study, men generally used a thinner water jet compared to that used by women. If the force of the water jet remains constant, a thin or narrow water jet may feel harder and more stimulating at the anus than a thick or wide water jet. Our previous study also showed that men, as opposed to women, used bidets more actively[3].

The bidet use score is an interview-led subjective assessment, and accurate physiological measurements of the bidet water jet, including its pressure, temperature, and thickness, were not made. Furthermore, the actual volume of water that may penetrate the rectum was not measured. These factors may explain why, in this study, the bidet use scores were not correlated with the FISI scores at baseline.

There are certain limitations to our study. First, this was a small retrospective study. The symptoms of AI or its severity may be affected by factors other than bidet use, such as the health status and physical limitations[14]. These were not evaluated in this study. Second, the findings of defecography and laboratory studies on anorectal function were not available in all patients. Furthermore, a risk of information bias cannot be ruled out as the patients were asked to report the symptom score, leading to possible over- or under- description of AI. Finally, the study population was selected from a single clinic in a tertiary care center, and our findings may not be generalizable to all patients with AI who use a bidet toilet.

In conclusion, symptoms of AI in patients who used electric bidet toilets to clean the anus significantly improved after the patients discontinued bidet use, suggesting that bidet use may be a possible cause of AI. If the patient uses a bidet, instead of treating AI immediately, the patient should be instructed to discontinue bidet use first. Further investigations should be made prospectively on whether people without the symptoms of AI who do not use an electric bidet toilet may experience AI after beginning bidet use.

\section{Acknowledgements}

The authors thank Yuko Tsunoda for her assistance with statistical analyses. 
Conflicts of Interest

There are no conflicts of interest.

\section{Author Contributions}

Akira Tsunoda: acquisition of data, analysis and interpretation of data, drafting of the article, and final approval of the version to be published.

Hiroshi Kusanagi: acquisition of data analysis and interpretation of data, critical revision of the article for important intellectual content, and final approval of the version to be published.

Approval by Institutional Review Board (IRB)

Institutional review board: Ethical Committee of Kameda Medical Center Review board approval number: 20-091

\section{References}

1. Manjoo F. Electronic bidet toilet seat is the luxury you won't want to live without. New York Times. Available from: https://www.nyti mes.com/2015/04/30/technology/personaltech/electronic-bidet-toile t-seat-is-the-luxury-you-wont-want-to-live-without.html. Retrieved Oct 14, 2020. 2015 Apr.

2. Cabinet Office, Government of Japan. Diffusion and home ownership of consumer durable goods (Japanese). 2019. Available from: https://www.esri.cao.go.jp/jp/stat/shouhi/shouhi.html/-Retrieved Oct 14, 2020.

3. Tsunoda A, Takahashi T, Arika K, et al. Survey of electric bidet toilet use among community dwelling Japanese people and correlates for an itch on the anus. Environ. Health Prev. Med. 2016 Oct; 21(6): 547-53.

4. Rockwood TH, Church JM, Fleshman JW, et al. Patient and surgeon ranking of the severity of symptoms associated with fecal incontinence: the fecal incontinence severity index. Dis Colon Rectum. 1999 Dec; 42(12): 1525-32.

5. Shorvon PJ, McHugh S, Diamant NE, et al. Defecography in nor- mal volunteers: results and implications. Gut. 1989 Dec; 30(12): 1737-49.

6. Bartram CI, Turnbull GK, Lennard-Jones JE. Evacuation proctography: an investigation of rectal expulsion in 20 subjects without defecatory disturbance. Gastrointest Radiol. 1988 Jan; 13(1): 7280.

7. Uchikawa K, Takahashi H, Deguchi G, Liu M. A washing toilet seat with a CCD camera monitor to stimulate bowel movement in patients with spinal cord injury. Am J Phys Med Rehabil. 2007 Mar; 86(3): 200-4.

8. Ryoo S, Song YS, Seo MS, et al. Effect of electronic toilet system (bidet) on anorectal pressure in normal healthy volunteers: influence of different types of water stream and temperature. J Korean Med Sci. 2011 Jan; 26(1): 71-7.

9. Cavanaugh M, Hyman N, Osler T. Fecal incontinence severity index after fistulotomy: a predictor of quality of life. Dis Colon Rectum. 2002 Mar; 45(3): 349-53.

10. Zutshi M, Tracey TH, Bast J, et al. Ten-year outcome after anal sphincter repair for fecal incontinence. Dis Colon Rectum. 2009 Jun; 52(6): 1089-94.

11. Wijffels NA, Jones OM, Cunningham $\mathrm{C}$, et al. What are the symptoms of internal rectal prolapse? Colorectal Dis. 2013 Mar; 15(3): 368-73.

12. Yagi Y, Tsunoda A, Takahashi T, Kusanagi H. Rectoanal intussusception is very common in patients with fecal incontinence. J Anus Rectum Colon. 2018 Oct; 2(4): 162-7.

13. Tsunoda A, Takahashi T, Yagi Y, Kusanagi H. Rectal intussusception and external rectal prolapse are common at proctography in patients with mucus discharge. J Anus Rectum Colon. 2018 Oct; 2 (4): 139-44.

14. Nelson R, Norton N, Cautley E, Furner S. Community-based prevalence of anal incontinence. JAMA 1995 Aug; 274(7): 559-61.

Journal of the Anus, Rectum and Colon is an Open Access journal distributed under the Creative Commons Attribution-NonCommercial-NoDerivatives 4.0 International License. To view the details of this license, please visit (https://creativ ecommons.org/licenses/by-nc-nd/4.0/). 